\title{
Augenheilkunde up2date
}

\section{$3 \cdot 2018$}

\section{Bindehaut, Hornhaut, Lederhaut 2}

\section{Moderne Hightech-Kontaktlinsen 2018 - mehr als nur ein Refraktionsausgleich}




\section{Unter dieser Rubrik sind bereits erschienen:}

Mikrobielle Keratitis - Teil 2: mykotische und Akanthamöbenkeratitiden I. Schmack, M. Müller, T. Kohnen Heft 1/2018

Mikrobielle Keratitis, Teil 1: allgemeine Aspekte und bakterielle Keratitiden I. Schmack, M. Müller, T. Kohnen Heft 1/2018

Diagnostik und Therapie von Irisläsionen J. M. Mor, K. R. Koch, L. M. Heindl Heft 4/2017

Nicht infektiöse Bindehauterkrankungen K. Schröder, D. Finis, S. Meller, B. Buhren, M. Wagenmann, G. Geerling Heft 2/2016

Diagnostik von Binde- und Hornhauterkrankungen K. Spaniol, R. Guthoff, S. Schrader, M. Borrelli, N. Kajasi, M. Schramm,

G. Geerling Heft 2/2016

Nicht infektiöse Bindehauterkrankungen T. Meyer-ter-Vehn Heft 1/2016

Diagnostik von Binde- und Hornhauterkrankungen D. Finis, P. Ackermann, S. Schrader, G. Geerling Heft 4/2015

Diagnostik von Binde- und Hornhauterkrankungen M. Roth, S. Schrader, D. Finis, K. Spaniol, C. MacKenzie, P. Ackermann, G. Geerling Heft 2/2015

Augenverätzungen N. Schrage Heft 2/2015

Therapeutische Hornhautchirurgie C. Meltendorf, N. Torun, H. Thieme Heft 1/2015
Episkleritis und Skleritis B. Sobolewska, M. Zierhut Heft 1/2015

Hornhaut: endotheliale Dystrophien, endotheliale Degenerationen T. Fuchsluger, W. Lisch, G. Geerling, B. Seitz Heft 4/2014

Diagnostik von Binde- und Hornhauterkrankungen S. Schrader, D. Finis, K. Spaniol, M. Roth, P. Ackermann, G. Geerling Heft 3/2014

Transforming-Growth-Factor- $\beta$-induzierte Dystrophien der Hornhaut D. Finis, J. Stammen, G. Geerling Heft 2/2014

Keratitis durch Herpes-simplex- und Varizella-zoster-Virus C. Tappeiner, A. Heiligenhaus Heft 1/2014

Bindehautinfektionen P. Steven, L. Heindl Heft 3/2013

Bindehautdegenerationen und -neoplasien C. Auw-Hädrich, L. Bredow, T. Reinhard Heft 2/2013

Sterile Keratitis E. Messmer Heft 1/2013

Epitheliale Dystrophien und Degenerationen der Hornhaut D. Finis, J. Stammen, G. Geerling Heft 1/2013

Keratokonus: Klinik, Diagnostik, Therapiemöglichkeiten J. Bühren, G. Bischoff, T. Kohnen Heft 1/2011

\section{ALLES ONLINE LESEN}

Mit der eRef lesen Sie Ihre Zeitschrift: online wie offline, am PC und mobil, alle bereits erschienenen Artikel. Für Abonnenten kostenlos! https://eref.thieme.de/augen-u2d

\section{JETZT FREISCHALTEN}

Sie haben Ihre Zeitschrift noch nicht freigeschaltet? Ein Klick genügt: www.thieme.de/eref-registrierung 


\section{Moderne Hightech-Kontaktlinsen 2018 - mehr als nur ein Refraktionsausgleich}

Gudrun Bischoff

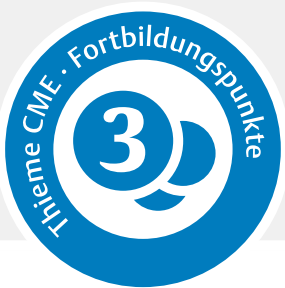

Kontaktlinsen gibt es schon seit mehr als 100 Jahren. Aber erst in den letzten Jahren hat die moderne Kontaktlinsenherstellung durch die Entwicklung neuer Materialien und Technologien die Indikationen stark erweitert. Ein Hot Topic mit weltweiter Beachtung ist die Prävention der fortschreitenden Myopie durch Einsatz speziell geformter Kontaktlinsen. Inzwischen werden Kontaktlinsen mit medizinischen Messgeräten kombiniert, was den Rahmen des reinen Refraktionsausgleichs sprengt.

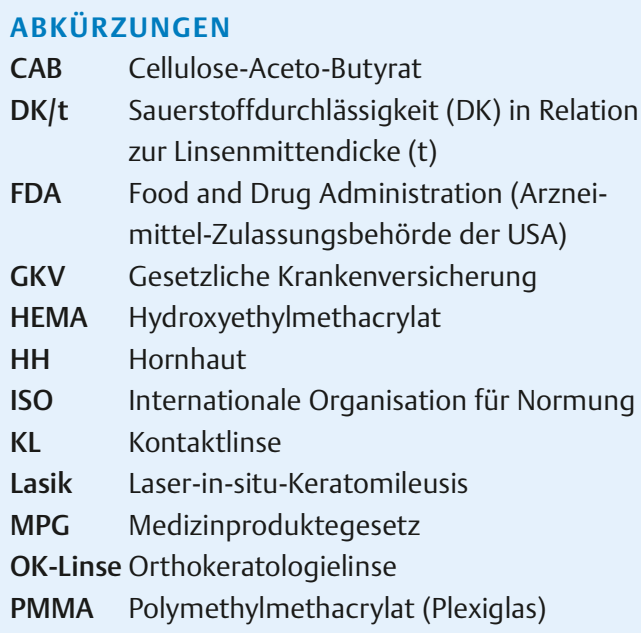

\section{Kontaktlinsentypen - Basiseinteilung}

Kontaktlinsen bestehen chemisch aus sog. inerten, biochemisch inaktiven Kunststoffen. Die Grobeinteilung definiert sich nach der Verformbarkeit: formstabil und weich ( $\bullet$ Abb. 1). Nach den ersten Kontaktlinsen aus Glas formte man formstabile Kontaktlinsen aus dem biologisch verträglichen Material PMMA, das ist Plexiglas. Diese Linsen waren aber sauerstoffundurchlässig, der Hornhautstoffwechsel war deprimiert. Nach einigen Stunden Tragezeit entstand ein Hornhautödem [1].

Die nächste Stufe in der Entwicklung waren Kontaktlinsen aus Cellulose-Aceto-Butyrat ( $\mathrm{CAB}$ ), mit geringer Sauerstoffdurchlässigkeit, die längere Tagestragezeiten zulieBen [2]. Weitere Zusatzstoffe wie z. B. Halogene verbesserten den Gasaustausch. Inzwischen hat jeder Material-
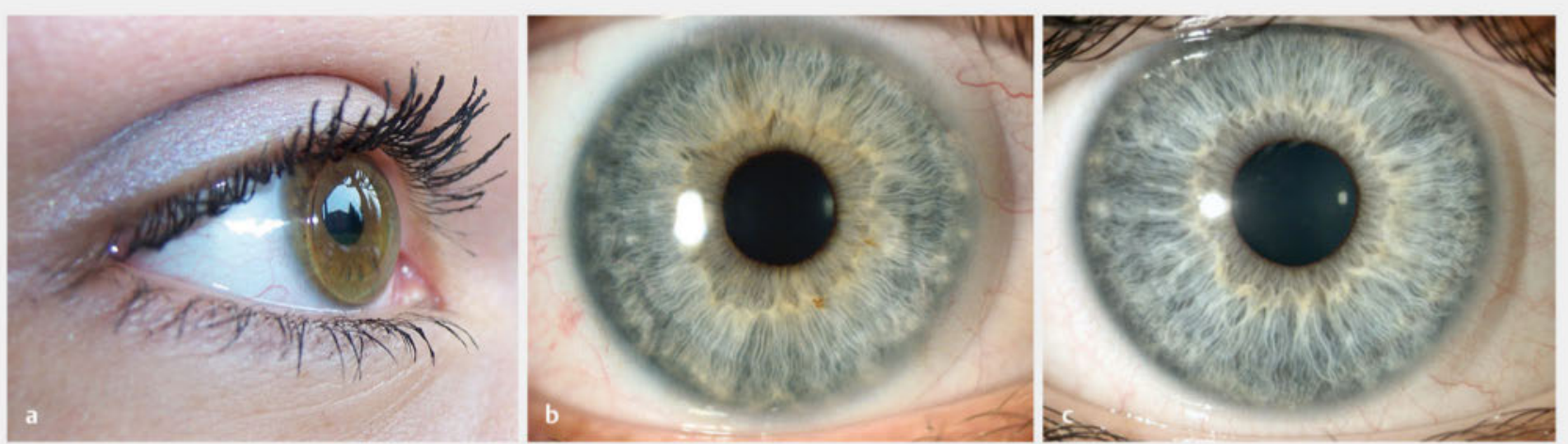

- Abb. 1 Historische Entwicklung der Kontaktlinsentechnologie.

a Formstabile Kontaktlinsen.

b Große weiche Kontaktlinsen.

c Silikon-Hydrogel-Kontaktlinsen. 
hersteller sein eigenes Rezept, um die optimale Balance zu erreichen zwischen Stabilität der Linsen und physiologischer Verträglichkeit [2].

Die ersten weichen Kontaktlinsen sind in den frühen 1970er-Jahren entwickelt worden, ein bekannter Name ist Otto Wichterle [3]. Die Träger forderten mehr Tragekomfort. Diese ersten Linsen aus HEMA (Hydroxyethylmethacrylat) hatten einen guten Komfort, aber erhebliche Nebenwirkungen. Die Augen wurden nach einigen Stunden rot und es bildeten sich über die Zeit limbusnahe Gefäßeinsprossungen. Auch hier hat die weitere Forschung und Entwicklung dazu geführt, dass die chemische Zusammensetzung der Weichlinsenkunststoffe genau die Vielfalt erreichte, die uns heute eine abge- stimmte individuelle und vor allen Dingen gesunde Anpassung ermöglicht [4].

Die nächste bahnbrechende Stufe in der Kontaktlinsenentwicklung waren die Silikon-Hydrogel-Linsen. Sie sind zwar flexibel, aber etwas steifer als die klassischen Hydrogellinsen. Durch den Anteil an Silikon haben diese Linsen eine sehr gute Sauerstoffdurchlässigkeit [5]. Die Tagestragezeiten haben sich dadurch deutlich verlängert [6], manche Linsen dürfen auch über Nacht als sog. „continous (extended) wear“ (Dauertragelinsen) getragen werden.

Eine Übersicht über die Charakteristika von Kontaktlinsen fasst $>$ Tab. 1 zusammen.

- Tab. 1 Charakteristika von Kontaktlinsen.

\begin{tabular}{|c|c|}
\hline Kontaktlinsenparameter & Daten auf der Box und im Herstellerkatalog \\
\hline Materialeinteilung & formstabile (harte) und weiche Linsen \\
\hline Radius/Basiskurve & $\begin{array}{l}\text { Rückflächenkrümmung der Kontaktlinse gemessen in mm, } \\
\text { abhängig vom Linsentyp }\end{array}$ \\
\hline Durchmesser & je nach Linsentyp von 8,00-25,0 mm \\
\hline Randabflachung der Linse - angepasst an die Form der Hornhaut & $\begin{array}{l}\text { Maßeinheit numerische Exzentrizität } \varepsilon \text {, das ist die Abweichung von der } \\
\text { Kreisform mit } \varepsilon 0 \text {, normale Hornhaut } 0,5\end{array}$ \\
\hline sphärische Stärke/Brechwert & $\begin{array}{l}-30,0 \mathrm{dpt} \text { bis }+30,0 \mathrm{dpt} \text { bei ausgewählten Herstellern, in der Regel bis } \\
\pm 12 \mathrm{dpt}\end{array}$ \\
\hline zylindrische Stärke & sehr hoch möglich, individuell bis 15,0 zyl dpt \\
\hline Achsenlage & $\begin{array}{l}\text { häufig in Schritten von } 5^{\circ} \text { oder } 10^{\circ} \text {, bei individuellen Linsen auch } \\
\text { in } 1^{\circ} \text {-Schritten }\end{array}$ \\
\hline Frontoptikzone & $\begin{array}{l}\text { Größe der korrigierenden zentralen Fläche, abgestimmt auf Stärke der } \\
\text { Linse und Randgestaltung }\end{array}$ \\
\hline Innenoptikzone = Geometrie der Linsenrückfläche & $\begin{array}{l}\text { Areal, welches innen der Hornhaut i. d. R. parallel aufliegt, } \\
\text { u. a. verantwortlich für den Sitz der Linse }\end{array}$ \\
\hline Stabilisierungsprinzip bei torischen Kontaktlinsen & $\begin{array}{l}\text { prismatisch und/oder dynamisch, abzulesen in der Beschreibung } \\
\text { des Herstellers }\end{array}$ \\
\hline $\begin{array}{l}\text { Wassergehalt } \\
\text { (indirekter Parameter für Komfort und Gasdurchlässigkeit, } \\
\text { hoher Wassergehalt = flexible Linsen) }\end{array}$ & moderne Materialien haben ein hohes Wasserbindungsvermögen \\
\hline $\begin{array}{l}\text { Sauerstoff-/Gasdurchlässigkeit } \\
\rightarrow \text { wichtig für die physiologische Verträglichkeit }\end{array}$ & $\begin{array}{l}\text { DK = Materialkonstante pro definiertem Areal } \\
\text { DK/t-Transmissibilität } \\
\text { Wert unter Einbeziehung der Dicke der jeweiligen Kontaktlinse, } \\
\text { Einheit gemessen bei Linsen - } 3,0 \mathrm{dpt} \\
\text { Wert ab 100: sehr gute Sauerstoffversorgung }\end{array}$ \\
\hline Einteilung der Materialien nach FDA & $\begin{array}{l}\text { FDA Gruppe I-IV: Klassifikation nach Wassergehalt, elektrischer } \\
\text { Oberflächenspannung } \\
\text { Zuordnung nach ISO-Standard }\end{array}$ \\
\hline bei multifokalen Linsen Größe und Sitz des Nah- und Fernzusatzes & nach Herstellerinformation, vielfältige Variationen möglich \\
\hline $\begin{array}{l}\text { Nutzungszeit der Kontaktlinse/Kontaktlinsentyp, Kennzeichnung } \\
\text { auf der Box }\end{array}$ & Tageslinse, Monatslinse, Multifokallinsen, torische Linsen etc. \\
\hline CE-Kennzeichnung mit Angabe einer Zahl & $\begin{array}{l}\text { Für den Vertrieb nach dem Medizinproduktegesetz (MPG) in Europa } \\
\text { zwingend vorgeschrieben. Die Zahl unter dem CE-Zeichen ist der Code für } \\
\text { die sog. Benannte Stelle, welche die Prüfung vorgenommen und das } \\
\text { CE-Zeichen vergeben hat }\end{array}$ \\
\hline $\begin{array}{l}\text { Verfalldatum } \\
\text { Chargen-/Lot-Nummer }\end{array}$ & $\begin{array}{l}\text { Werte auf der Box, die nach dem Medizinproduktegesetz (MPG) } \\
\text { bei Abgabe dokumentiert werden müssen }\end{array}$ \\
\hline
\end{tabular}




\section{Kontaktlinsen und Qualität}

In Europa dürfen nur Kontaktlinsen als Medizinprodukt mit der CE-Kennzeichnung des zertifizierten Prüflabors (benannte Stelle) vertrieben werden. Alle CE-gekennzeichneten Kontaktlinsen unterliegen einem geprüften und dokumentierten Herstellungsverfahren und sind für die erklärten Indikationen geeignet. Das erlaubt keine Aussage über die individuelle Verträglichkeit der Kontaktlinsen.

Die Auswahl des individuell geeigneten Linsentyps, hier am Beispiel der wasserhaltigen weichen Kontaktlinsen, wird erleichtert bei Kenntnis der Materialeigenschaften. Diese sind nach internationalen Standards (ISO 18369-1) eingeteilt. Aus der Gruppeneinteilung ( $\triangleright$ Tab. 2) lassen sich die Oberflächeneigenschaften ablesen. Eine ionische Oberfläche, also eine Oberfläche mit elektrischer Ladung, zieht mehr Ablagerungen an, deshalb sollte dieses Material bevorzugt für Kontaktlinsen zum kurzen Tragen, z. B. von Tageslinsen, eingesetzt werden.

Bei der Materialkomposition suchen die Hersteller das jeweilige Optimum zwischen Komfort, Stabilität, Reißfestigkeit, guter Handhabung und natürlich physiologischer Verträglichkeit und dadurch die Akzeptanz des Trägers.

Kontaktlinsen fallen nach dem MPG unter die Kategorie Implantate der Klasse 2, weil sie in das vordere Augensegment „eingebracht“ werden. Die Verträglichkeit ist deshalb zum einen definiert durch einen guten Visus, also die Funktion der Linse, aber auch durch ein gesundes, normal aussehendes und reizfreies Auge.

Die Abstimmung auf die individuellen Voraussetzungen des Trägers erfolgt nach folgenden Kriterien:

- Art des Refraktionsfehlers,

- anatomische Gegebenheiten des Auges (HH-Topografie),

- Tränenfilmstatus,

- Arbeitsumgebung,

- Vorerkrankungen

- eventuelle Medikamenteneinnahmen,

- gewünschte Tragezeit,

- Allergieneigung.

\section{Merke}

Durch die Erweiterung der Kontaktlinsenparameter aufgrund des technologischen Fortschritts ist es heute möglich, fast jeden Refraktionsfehler kontaktoptisch auszugleichen. Im Einzelfall sendet der Anpasser die Topografiedaten an den Hersteller, und der fertigt danach die Linse.
- Tab. 2 Einteilung der wasserhaltigen weichen Kontaktlinsen nach ISO 18369-1.

\begin{tabular}{|l|l|}
\hline Einteilung & Materialeigenschaften \\
\hline Gruppe I & unter $50 \% \mathrm{H}_{2} \mathrm{O}$ und nicht ionisch (<1\% ionische Monomere) \\
\hline Gruppe II & über $50 \% \mathrm{H}_{2} \mathrm{O}$ und nicht ionisch (<1\% ionische Monomere) \\
\hline Gruppe III & unter $50 \% \mathrm{H}_{2} \mathrm{O}$ und ionisch (> $1 \%$ ionische Monomere) \\
\hline Gruppe IV & über $50 \% \mathrm{H}_{2} \mathrm{O}$ und ionisch (> $1 \%$ ionische Monomere) \\
\hline
\end{tabular}

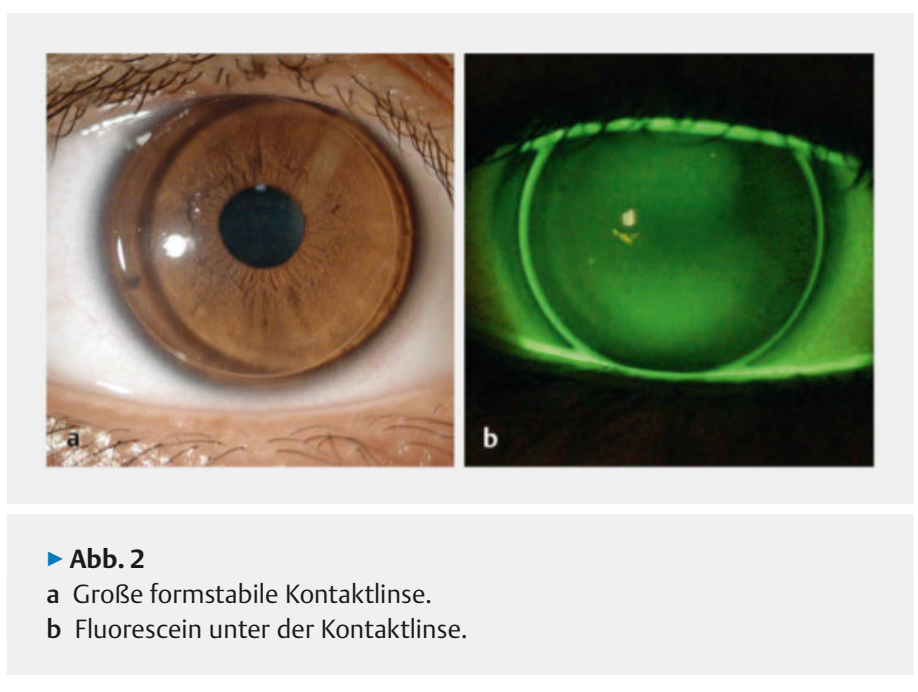

Eine Sitzkontrolle erfolgt bei formstabilen Linsen nach dem Fluoresceinbild unter der aufgesetzten Kontaktlinse, ferner unter Beurteilung der Bewegungs- und Zentriereigenschaften der Linsen. Einzelne Anpassprogramme der Hersteller simulieren auf dem Topografen dieses Bild. Die rechnerische Dicke des Tränenfilms unter der Linse wird farbkodiert dargestellt ( $\bullet$ Abb. 2). Dadurch wird die Anpassung virtuell und leichter und die Patientenzeit („chair time“) in der Praxis verkürzt.

\section{Die klassischen medizinischen Kontaktlinsenindikationen}

Im unkomplizierten kosmetischen Bereich werden die Kontaktlinsen je nach Refraktion und Tragewunsch ausgewählt. Für nur gelegentliches Tragen, z. B. für sportliche Aktivitäten, eignen sich besonders weiche Monatsoder Tageslinsen.

Bei den medizinisch-optischen Indikationen ist oft die Kontaktlinsenversorgung die einzige Möglichkeit, eine optische Rehabilitation zu erreichen.

Eine Übersicht über die Indikationen nach dem Hilfsmittelverzeichnis des GKV-Spitzenverbands zeigt $>$ Tab. 3. 
- Tab. 3 Indikationen nach dem Hilfsmittelverzeichnis des GKV-Spitzenverbands.

\begin{tabular}{|l|l|}
\hline Befund & Wert \\
\hline hohe Myopie & $\begin{array}{l}\text { ab 8dpt (nur für gesetzliche } \\
\text { Krankenkassen) }\end{array}$ \\
\hline hohe Hyperopie & $\begin{array}{l}\text { ab 8 dpt (nur für gesetzliche } \\
\text { Krankenkassen) }\end{array}$ \\
\hline Anisometropie & ab 2 dpt \\
\hline Aniseikonie & ab 7\% \\
\hline Aphakie & einseitig (wegen der hohen \\
\hline Astigmatismus rectus und inversus & Anisometropie) \\
\hline Astigmatismus obliquus & ab 3 dpt \\
\hline Irislinse bei Substanzverlust der Iris & ab 2 dpt \\
\hline klinische Definition hohe Myopie & ohne Stärke \\
\hline klinische Definition hohe Hyperopie & ab 6 dpt \\
\hline
\end{tabular}

\section{Okklusionslinsen}

Okklusionslinsen werden bevorzugt eingesetzt in der Schieltherapie. Grundsätzlich wird in der Literatur darauf hingewiesen, besonders Kinder frühzeitig voll mit Brillen oder Kontaktlinsen auszukorrigieren.

Dunkel gefärbte Kontaktlinsen dienen als wenig störender Okkluder in der Amblyopiebehandlung. Besonders bei Schulkindern werden sie gut akzeptiert. Man unterscheidet Kontaktlinsen mit schwarz gefärbter Pupille, die aber immer noch etwas Licht durchlassen. Ab einem Visus von 0,5 sind diese Linsen in der Amblyopiebehandlung brauchbar. Unter einem Visus von 0,5 empfiehlt es sich, Kontaktlinsen zu verwenden, die schwarz unterlegt und absolut lichtdicht sind. So kann das Kind mit völliger Unterdrückung des Seheindrucks aus der schweren Amblyopie geholt werden.

Für den Gebrauch der Okklusionslinsen werden die Eltern in der Handhabung geschult. Es erfolgen regelmäßige augenärztliche Kontrollen der Therapie.

\footnotetext{
Merke

Junge Patienten in der Amblyopiebehandlung müssen regelmäßig kontrolliert werden. Bei unsachgemäßer Anwendung der Okklusion besteht sonst die Gefahr einer induzierten Amblyopie auf dem okkludierten gesunden Auge.
}

Im Unterschied dazu reicht es bei störender Diplopie, das Auge zu vernebeln mit Linsen im hohen Plusbereich. Das hat auch den Vorteil, dass das periphere Gesichtsfeld erhalten bleibt, was Unsicherheiten beim Gehen vorbeugt und die Sturzgefahr mindert. Diese Therapie ist scho- nend, preiswert und effektiv für die meist älteren Menschen mit Diplopie nach Paresen.

\section{Farblinsen und Partylinsen}

Farbige Kontaktlinsen dienen primär kosmetisch der Umgestaltung der Augen. Sie werden häufig genutzt von jungen Damen und im Filmgewerbe. Eine beliebte Besonderheit sind Linsen mit dunklem Rand an der Iriszeichnung. Optisch wirkt das Auge groß. Seit wenigen Jahren sind farbige Kontaktlinsen auch als Hilfsmittel anerkannt und unterliegen in der Qualität den europäischen Richtlinien. Davor gab es erheblichen Missbrauch in Bezug auf Hygiene und Sorgfalt. Linsen wurden getauscht und „secondhand“ verkauft, mit allen bekannten Risiken.

Partylinsen sind bemalt zum Fasching und zu Halloween im Einsatz. Auch diese Linsen dürfen nicht von einem Träger zum nächsten gereicht werden.

\section{Cave \\ Partylinsenträger müssen in der Praxis besonders sorgfältig untersucht werden. Oft wird die Anamnese nicht vollständig angegeben, und es wurde doch ein Linsentausch vorgenommen. Vorsicht vor Über- tragung von Krankheitserregern bei nachlässiger Hygiene und Desinfektion!}

\section{Babylinsen}

Neugeborene mit einer deutlichen offensichtlich visusreduzierenden Katarakt werden sehr früh operiert. Innerhalb weniger Tage muss dann die Kontaktlinsenversorgung erfolgen, da sich sonst eine Amblyopie entwickelt. Geeignet sich Silikonlinsen mit einer extrem hohen Sauerstoffdurchlässigkeit von einem DK/t über 300.

Silikonlinsen werden kontinuierlich getragen und alle 3-4 Wochen gereinigt und ausgetauscht, wenn die Ablagerungen zu stark geworden sind. Das Silikon zieht Lipide an, die Linsen werden schlierig und lassen in der Abbildungsqualität nach. Silikonkontaktlinsen neigen auch dazu, sich festzusetzen, was wegen der hohen Sauerstoffdurchlässigkeit dieses Materials für kürzere Zeiten toleriert werden kann.

\section{TIPP}

Silikonlinsen gibt es nur von wenigen Herstellern, und sie haben längere Lieferzeiten, deshalb empfiehlt es sich immer, ein Reservepaar für die kleinen Patienten vorrätig zu halten.

Es gibt auch Babylinsen aus Silikon-Hydrogel, die aber eine etwas reduzierte Transparenz aufweisen. Babylinsen gehen in der Stärke bis +40 dpt, die Linsen sind dick. Es kommt auf den Einzelfall an, welcher Typ zum Einsatz kommen wird. 
Einzelne Anpasser besonders aus Deutschland, Frankreich und Holland bevorzugen wegen der guten Abbildungsqualitäten schon bei den Kleinsten hochgasdurchlässige formstabile Kontaktlinsen, die kontinuierlich über mehrere Wochen im Auge bleiben. Dies wird unter den Anpassern aber durchaus kontrovers diskutiert.

\section{Einteilung und Benennung der Kontaktlinsensysteme}

Die Literatur rund um Kontaktlinsen ist in der Begrifflichkeit vielschichtig und sogar verwirrend. Die Zuordnung der verschiedenen Kontaktlinsentypen und Designvarianten ist aber für die Anpassung und die Kontrolle wichtig. Die meisten Begriffe sind selbsterklärend und deskriptiv. Die Einteilungen können sich aber durchaus überschneiden. Eine Übersicht fasst $>$ Tab. 4 zusammen.

\section{Kontaktlinsen und Presbyopie}

Kontaktlinsenträger aus den frühen 1970er-Jahren kommen jetzt in das Presbyopiealter, und besonders diese Träger möchten keine Lesebrille tragen.

Presbyopiekontaktlinsen haben im Falle von Bifokallinsen einen doppelten Fokus, einen für die Nähe und einen für die Ferne, was die nachlassende Akkommodation entlastet $(\triangleright$ Abb. 3). Optisch wird das erreicht mit einer simul- tanen Anordnung von Ringen unterschiedlicher Stärke oder segmentaler Anordnung wie bei einer Brille. Bei den simultanen Linsentypen kann die Korrektur für die Nähe oder für die Ferne im Zentrum der Linse angeordnet sein. Auch gibt es diese beiden Varianten in multifokaler Ausführung, ähnlich einem Gleitsichtbrillenglas. Damit kann im Gegensatz zur Brille ein blickrichtungsunabhängiges Sehen in allen Entfernungsbereichen erreicht werden.

Möglich ist die Fernkorrektur zentral an einem Auge, das zweite Auge hat eine zentrale Nahkorrektur, ausgewählt je nach dominantem Auge und für den entsprechenden gewünschten Arbeitsabstand.

Für Presbyopielinsen ist der binokulare Seheindruck entscheidend.

\section{TIPP}

Diese Linsen haben alle mehr oder weniger ausgeprägte optische Nebenwirkungen wie z.B. Halos. Deshalb erfordert diese Anpassung einen kooperierenden und motivierten Träger. Er muss seine individuelle Linsenkonfiguration verstehen und den erreichten Sehkomfort akzeptieren [9]. Die klinische Erfahrung zeigt, dass anderenfalls die Anpassung von Presbyopielinsen nicht erfolgreich ist.

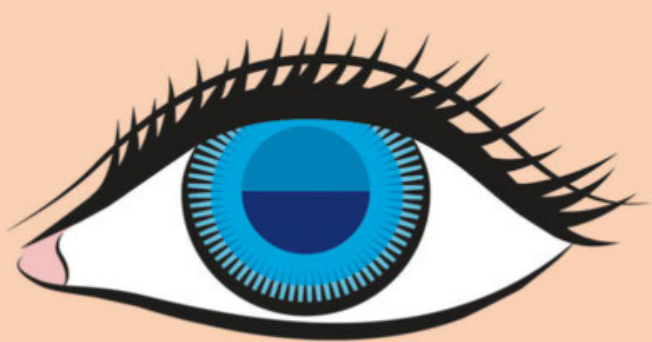

a

Abb. 3 Prinzipien der Presbyopielinsen.

a Segmentale bifokale Kontaktlinse.

b Simultane multifokale Kontaktlinse

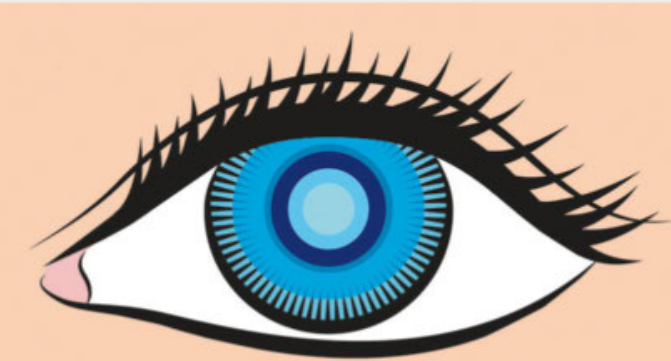

b 
- Tab. 4 Begrifflichkeiten im Zusammenhang mit Kontaktlinsen.

\section{Gruppeneinteilung/Überbegriff}

Material/Linsenart

Einteilung nach Größe

kosmetisch-ästhetisch

Design

(wird benannt je nach Form der Vorder- und Rückfläche, wobei fast alle möglichen Kombinationen lieferbar sind)

Keratokonuskontaktlinsen

\section{Tragezeit}

(richtet sich nach den Materialeigenschaften und der Sauerstoffdurchlässigkeit)

Austauschfrequenz

Sauerstoffdurchlässigkeit - Gasaustausch

Wassergehalt

Parameter und Lieferumfang

Therapielinsen (Verbandlinsen)

Kontaktlinsen zur operativen Nachbehandlung

Beschreibung der Indikationen

\section{Bezeichnung}

- formstabil

- flexibel

- weich

- Hydrogellinsen

- Silikon-Hydrogel-Linsen

- Silikonlinsen

- korneale Kontaktlinsen, die kleiner sind als der Hornhautdurchmesser - bis ca. 10 mm

- semisklerale Kontaktlinsen, die gerade über den Limbus reichen - bis 14 mm

- Sklerallinsen - bis zu 25 mm alle für den Träger verträgliche Linsentypen, je nach Wunsch

rotationssymmetrisch, sphärisch oder asphärisch, torische nicht rotationssymmetrische KL wie z. B. rücktorisch, periphertorisch, vorderflächentorisch, quadrantenspezifisch bi- oder multifokale KL simultan, segmental

Sonderlinsen wie z. B. Hybridkontaktlinsen = fester zentraler Bereich und weicher Rand

meist genutzt werden formstabile Linsen, die exakt den Konus nachformen; es gibt aber auch weiche spezielle Keratokonuslinsen [7]

- Tageslinsen = zum Tragen über den Tag

- Linsen zum flexiblen Tragen = gelegentlich auch über die Nacht

- Dauertragelinsen („,continous/extended wear“) = bis 30 Tage ohne Austausch

- Tageslinsen

- Monatslinsen

- Dreimonatslinsen

- Jahreslinsen

Hypergasdurchlässigkeit mit einer Transmissibilität von DK/t > 125, hochgasdurchlässig 80-125, mittelgasdurchlässig 30-80, nicht/wenig gasdurchlässig 30

wird in \% angegeben

je höher der Wassergehalt, desto weicher und fragiler die Linse; Wassergehalt

- bei formstabilen KL 0-5\%

- bei weichen KL 24-85\%

- konventionell - mit definierten vorgegebenen Parametern

- individuell - nach den Daten des Trägers gefertigt

zur Heilung oberflächlicher Hornhautverletzungen - alle weichen Kontaktlinsen, die aufgrund der hohen Sauerstoffdurchlässigkeit verlängert getragen und eine Zulassung als Verbandlinse haben

in der Regel weiche Therapielinsen

Ausnahme: steifere Silikon-Hydrogel-Linsen zur Glättung einer oberflächlichen Topografie nach z. B. Keratoplastik

- klassische Kontaktlinsen

- Babykontaktlinsen

- Farblinsen/Sonnenschutzlinsen

- Kontaktlinsen zur Myopieprävention

- Presbyopiekontaktlinsen

- Keratokonuslinsen

- Okklusionslinsen zum Abdecken eines Auges

- Partylinsen

Infektionsrisiko

je nach Material und Tragezeit:

- das geringste Risiko haben die formstabilen Kontaktlinsen im Tagestragen

- das höchste Risiko von infektiösen Komplikationen haben die weichen Dauertragelinsen mit einer Komplikationsrate von 28,9/10000 [8]

Kontaktlinsen als Medikamententräger am besten geeignet: Kontaktlinsen mit großen Poren und hohem Wassergehalt 


\section{FALLBEISPIEL}

\section{Fall 1: Wunsch nach Kontaktlinsenanpassung}

Die Patientin, Ende 40, arbeitet im Büro, ständig am PC, schreibt nach Diktat und wenig nach Vorlagen. Sie wünscht keine Lesebrille.

Es liegt keine Kontaktlinsenkontraindikation vor. Zuerst erfolgt die Überprüfung der Kontaktlinsenmotivation. $\mathrm{Bi}$ - und multifokale Kontaktlinsen trennen den Seheindruck und das Gehirn selektiert. Dadurch ist der einzelne Bereich etwas schlechter als bei monofokalen Linsen. Bei manchen Patienten erfordert das eine gewisse Eingewöhnungszeit, darüber wird aufgeklärt.

Ist es wichtig, die Sicht im mittleren Arbeitsabstand zum PC zu erleichtern, dann bieten sich als erste Maßnahme Kontaktlinsen mit aplanatischer Abflachung zum Rande hin an. Wird diese ursprüngliche optisch fehlerhafte $A b-$ bildung einer Linse nicht mathematisch korrigiert, dann hat die Linse mehrere Brennpunkte. Dieser multifokale Effekt wird genutzt, er erleichtert das Sehen in verschiedenen Abständen. Das Auge sucht, zerebral gesteuert, selektiv den richtigen Fokus aus. Störende weitere Bilder werden unterdrückt.

Linsen mit reinem aplanatischem Design werden angepasst wie einfache sphärische Linsen und sind für Jungpresbyope sehr gut in der Verträglichkeit.

Wünscht die Patientin eine ständige Korrektur der Presbyopie, kommen multifokale Kontaktlinsen infrage. Die
Auswahl geht über eine genaue Bestandsaufnahme der Sehwünsche. Wird lange am PC gearbeitet, dann muss der mittlere Fokalbereich möglichst groß sein, man wählt eine PC-Kontaktlinse. Wird daneben viel im nahen Bereich gearbeitet, werden Vorlagen gelesen und korrigiert, darf der additive Nahzusatz nicht zu gering und zu klein ausfallen. In Abhängigkeit von der Binokularität gibt es die Auswahl mit unterschiedlichen Additionen und unterschiedlichen Größen der einzelnen Bereiche. Außerdem zu beachten ist die Weite der Lidspalte, da manche Kontaktlinsen sich beim Blick nach unten erst in die richtige Position schieben.

Letztendlich ist jeder Patient gut zu versorgen. Schon diese Aufzählung macht aber deutlich, dass bei der Versorgung mit einer Presbyopielinse der Anpasser seinen bevorzugten Linsenhersteller und dessen Linsen gut kennen muss, der Träger muss seine Wünsche ausdrücken können und verständig sein.

\section{Fazit für die Praxis}

Bei jüngeren Patienten um die 42+ Jahre immer nach einem Kontaktlinsenwunsch zur Presbyopiekorrektur fragen. Oft bestehen bei Jungpresbyopen hierzu Schwellenängste.

\section{Kontaktlinsen bei Keratokonus}

Keine andere Augenerkrankung ist in den letzten Jahren häufiger diskutiert worden als der Keratokonus. Das hat verschiedene Gründe. Einmal tritt der Konus als progressiv ektatische Erkrankung der Hornhaut ethnisch gehäuft auf bei jungen Menschen aus dem östlichen Mittelmeerraum und dem mittleren Osten. Diese klinische Erfahrung macht jeder Anpasser. Andererseits gibt es inzwischen mit der exzellenten Kontaktlinsenversorgung und dem Cross-Linking bis hin zu den verschiedenen Methoden der operativen Hornhautversorgung sehr erfolgreiche und gute Behandlungsmethoden.

Durch die Gewebeschwäche beim Keratokonus kommt es zu einer Vorwölbung der Hornhaut:

- zentral in leichten Fällen,

- vorwiegend versetzt nach nasal unten bei stärkerer Ausprägung.

Die Therapieoption der ersten Wahl ist die Kontaktlinsenversorgung mit formstabilen Linsen. Einfach gestaltet sich dies bei zentrischen kleinen runden Koni. Erleichtert wird die Keratokonusanpassung mit den verfügbaren
EDV-Programmen der Hersteller, welche den Linsensitz simulieren und über eine mathematische Berechnung der Dicke des Tränensees ein simuliertes Fluoresceinbild erstellen.

Eine Optimierung des KL-Sitzes kann ebenfalls virtuell durch Veränderung der Basiskurven, Exzentrizitäten und Durchmesser vorgenommen werden, bis nach dem Fluoresceinbild der Linsensitz passend erscheint. So hat die moderne EDV-gestützte Anpassung auch diese schwierigen Versorgungen deutlich verbessert und erleichtert ( Abb. 4).

\section{Besondere \\ Kontaktlinsenversorgungen}

Nach einer Lasik-Operation mit den Geräten einer frühen Generation können manchmal späte sekundäre Ektasien auftreten (s. a. Fallbeispiel 2). Darunter versteht man eine kegelartige Vorwölbung der Hornhaut aufgrund fehlender Stabilität [10]. Diese Komplikation, die in der Art und Ausprägung dem idiopathischen Keratokonus gleicht, wird genau wie dieser behandelt. Bei geringer Ausprä- 

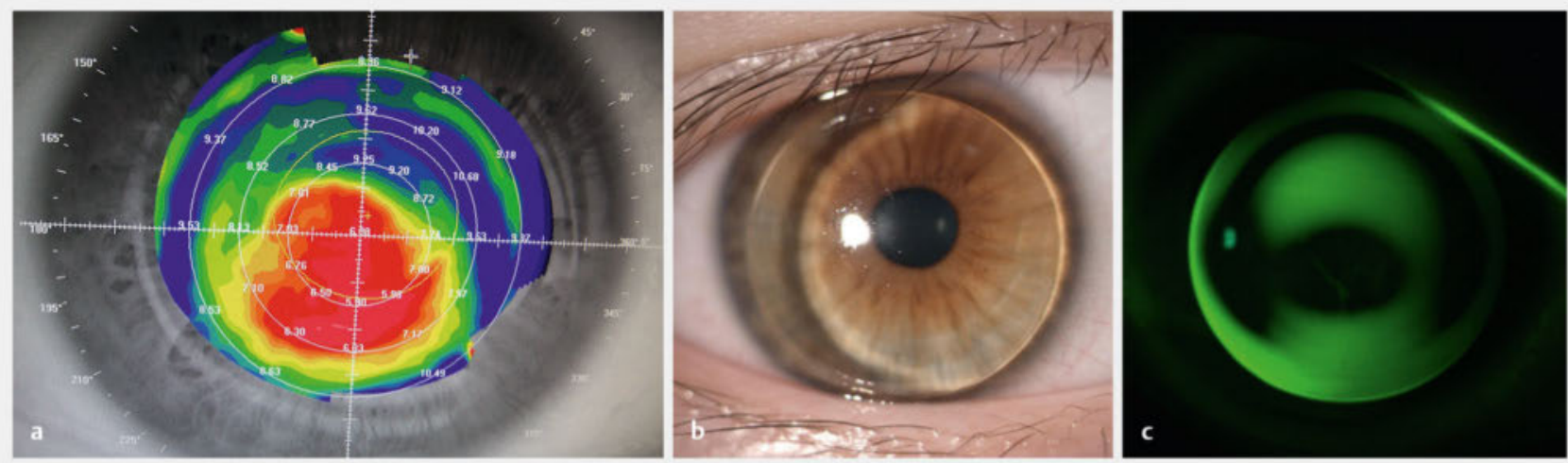

- Abb. 4 Kontaktlinsenanpassung bei Keratokonus.

a Topografie des Keratokonus.

b Keratokonuslinse mit leicht exzentrischem Sitz.

c Fluoresceinbild eines Keratokonus.

gung reicht die Anpassung von formstabilen Kontaktlinsen. Bei stärkerer Ausprägung führt der Ophthalmochirurg ein Cross-Linking (s. Definition) durch. Nach Abheilung und Epithelschluss werden Kontaktlinsen angepasst.

\section{DEFINITION}

\section{Ophthalmochirurgisches Cross-Linking}

Biochemische Versteifung der Kornea durch Quervernetzung der Kollagenfibrillen unter $\mathrm{B}_{12}$-Benetzung und Bestrahlung mit UV-Licht. Vorübergehend wird dadurch die Keratozytenzahl vermindert.

\section{TIPP}

Neue Studien zeigen, dass es in Einzelfällen sinnvoll sein kann, schon während der Lasik-Prozedur ein prophylaktisches Cross-Linking durchzuführen [11].

Sekundäre Ektasien findet man auch nach perforierender Keratoplastik aufgrund eines Keratokonus. Nach ca. 10 Jahren übernimmt das Transplantat die pathologische Hornhautform. Hier erfolgt die Behandlung nach dem gleichen Schema mit Cross-Linking und nachfolgender Kontaktlinsenversorgung [12].
Eine weitere Kombination von Kontaktlinsen und Operation ist die Kontaktlinsenanpassung nach halbmondförmiger kornealer Segmentimplantation beim Keratokonus. Hier wird erst die ektatische Topografie durch die intrakornealen Ringe angehoben, ggf. kann zur weiteren Stabilisierung ein Cross-Linking aufgesetzt werden. Dann erfolgt die Kontaktlinsenkorrektur. Oft ist in der Folge nur eine Kontaktlinse mit einfacher Geometrie notwendig [13].

Einfache glatte perforierende Verletzungen werden schon seit Langem behandelt, indem über eine gewisse Zeit eine gut verträgliche Therapielinse aufgesetzt wird. Diese adaptiert die Wundränder, es entsteht nur eine zarte strichförmige Narbe. Diese Bandagenwirkung durch die Linse nutzt auch der Chirurg, falls es nach KataraktOP zu Schnittdehiszenzen kommt. Auch nach filtrierender Glaukomoperation kann die Höhe und die Größe des Filterkissens mit einer großen bandagierenden Therapielinse reguliert werden.

\section{TIPP}

Die Kombination von Kontaktlinsen zur Unterstützung der Heilung, zur Schmerzlinderung und zur schnellen Rehabilitierung der Patienten und zur Problemlösung nach operativen Eingriffen erfordert ärztliche Kreativität, kontaktoptische Kenntnisse und eine gute kollegiale Zusammenarbeit. 


\section{FALLBEISPIEL}

\section{Fall 2: Visusverschlechterung bei Z. n. Lasik}

Der Patient ist Mitte 40, hatte eine Lasik vor 20 Jahren und stellt sich jetzt vor, weil die Sehleistung sich in den letzten Jahren verschlechtert.

\section{Genaue Anamnese}

Welche Art der Behandlung wurde durchgeführt? Wie umfangreich war die präoperative Untersuchung?

\section{Diagnostik}

Die aktuelle Diagnostik erfolgt idealerweise mit einer Scheinpflug-Kamera unter Einbeziehung der inneren Hornhauttopografie.

Gründe gibt es viele, wenn sich nach Jahren eine sekundäre vordere Ektasie ausbildet. Heute beachtet man neben der Hornhautdicke auch die Rigidität der Hornhaut. Danach wird die Ablationstiefe bestimmt. An der Struktur der inneren Hornhaut kann ein früher Keratokonus, eine „forme fruste“, diagnostiziert werden.

\section{Therapie}

Die Behandlung erfolgt, wenn möglich und die Hornhaut noch eine restliche Dicke von mindestens $400 \mu \mathrm{m}$ aufweist, klassisch mit Cross-Linking und anschließender Kontaktlinsenversorgung.

\section{Fazit für die Praxis}

Nicht immer ist eine jahrzehntealte Ablationszone gut zu sehen. Deshalb bei Hinweis oder Verdacht auf eine frühere Lasik-Behandlung sorgfältige Spaltlampenkontrolle vornehmen.

\section{Orthokeratologie}

Zwischen der refraktiven Chirurgie und dem täglichen Tragen von Kontaktlinsen ist die Orthokeratologie angesiedelt. Man nennt diese Linsen auch OK-Linsen oder „Nachtlinsen“. Die formstabilen OK-Linsen werden nur stundenweise im Schlaf getragen. Durch eine Abflachung im zentralen Bereich verformen sie die Hornhaut um ca. $30 \mu \mathrm{m}$ und verändern so deren Brechkraft ( $>$ Abb. 5). Man nutzt diesen sog. Ortho-K-Effekt für Myopien bis ca. 4-6 dpt, je nach Hersteller und Linsentyp. Bei höheren Dioptrienwerten wäre der zentrale abgeflachte Bereich zu klein bzw. kleiner als die mittlere Pupillenweite. Lichtreflexe durch Brechung am Rande der komprimierten Zone werden dann als sehr störend empfunden.
Beim OK-Träger ist eine Refraktionskorrektur über den Tag nicht mehr nötig. Dieser Emmetropieeffekt hält für 8-12 Stunden. Wenn darüber hinaus noch volle Sehkraft gewünscht wird, trägt der Patient seine OK-Linsen, die ihn voll korrigieren.

Studien haben gezeigt, dass nach völligem Absetzen der OK-Linsen die Hornhaut wieder ihre ursprüngliche Form annimmt. Diese Linsen schaden dem Auge und seinen Strukturen nicht.

\section{Myopie und Myopieprävention}

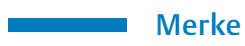

Eine neue interessante Indikation für die OK-Linsen ist die Myopieprävention [14].

Ein wichtiges Gebiet für die medizinische Kontaktologie eröffnet sich mit der Myopieforschung. Die hohe Myopie ist als Krankheit einzustufen, sie birgt das Risiko schwerwiegender Netzhautveränderungen. So ist bei hoher Myopie die Amotiorate erhöht, es finden sich vermehrt Makulaschäden und Degenerationen. Da besonders in Asien die Myopierate der Bevölkerung schon bei knapp $90 \%$ liegt, kommt ein großer Teil der Forschung und der Studien aus dieser Region.

Die Entstehung der Myopie ist einmal genetisch bedingt, Umweltfaktoren spielen ebenso eine große Rolle. So fördert die exzessive Naharbeit und der ständige Blick auf Smartphone und Tablet die Progression. Messbar ist dies über eine Zunahme des Längenwachstums des Bulbus ( Abb. 6).

Ein Trigger für das zunehmende Längenwachstum des Bulbus ist $u$. a. die relative periphere Hyperopie bei einer einfachen sphärischen Brillen- oder Kontaktlinsenkorrektur. Auskorrigiert wird ein Patient immer mit der Fokalebene in der Makula. Der Fokus für die peripheren Netzhautareale liegt dann hinter dem Bulbus. Das regt auf humoralem Weg und interne Rückkopplung das Augenwachstum an, der Bulbus elongiert und wird stärker myop. Stoppen kann man dieses Längenwachstum mit speziellen Kontaktlinsen mit peripherer Addition. Die periphere Fokalebene wird auf die Netzhaut projiziert, der Wachstumsreiz entfällt.

\footnotetext{
Merke

Das Bulbuswachstum kann durch Linsen mit peripherer Pluswirkung gestoppt werden.
}

Je nach Hersteller gibt es dafür unterschiedliche und sehr aufwendige Designvarianten [15]. 


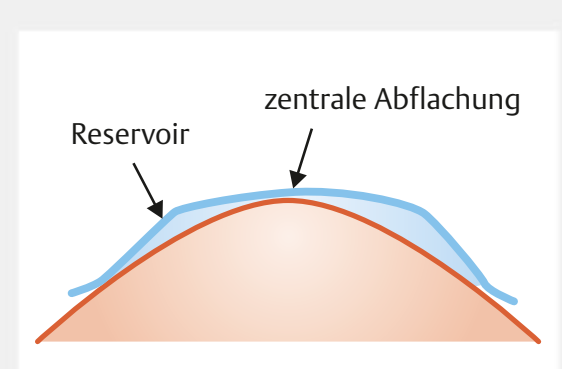

a
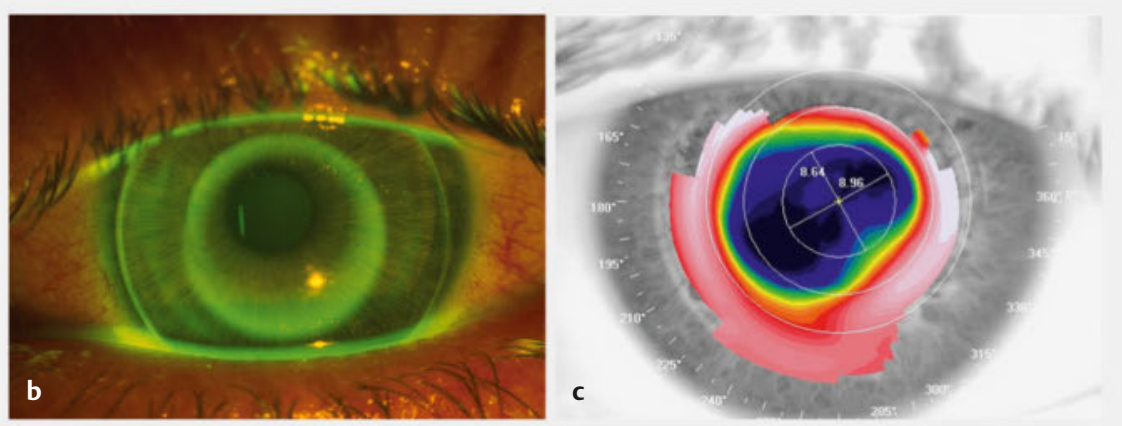

\section{SZOCULUS:}
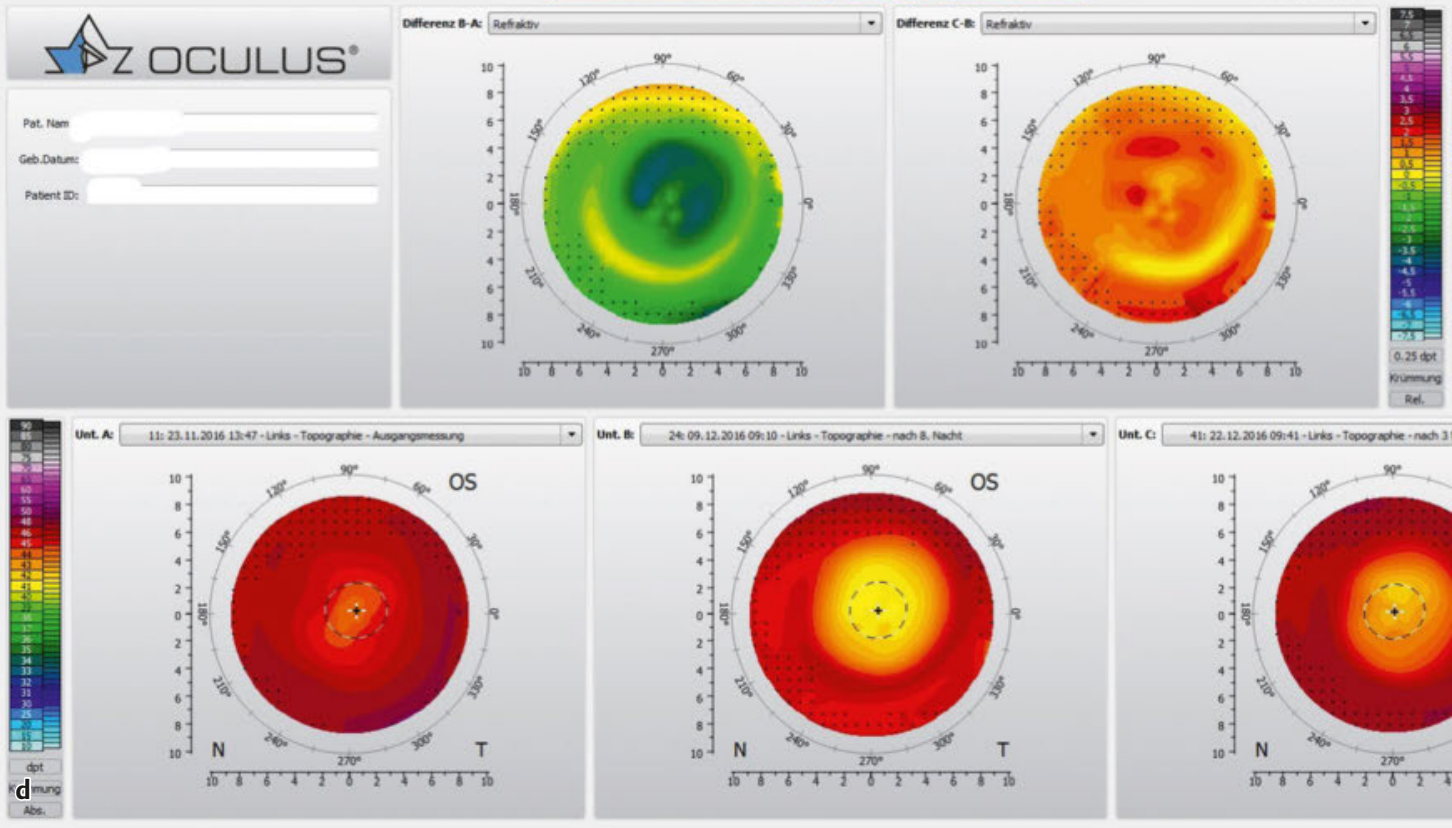

- Unt
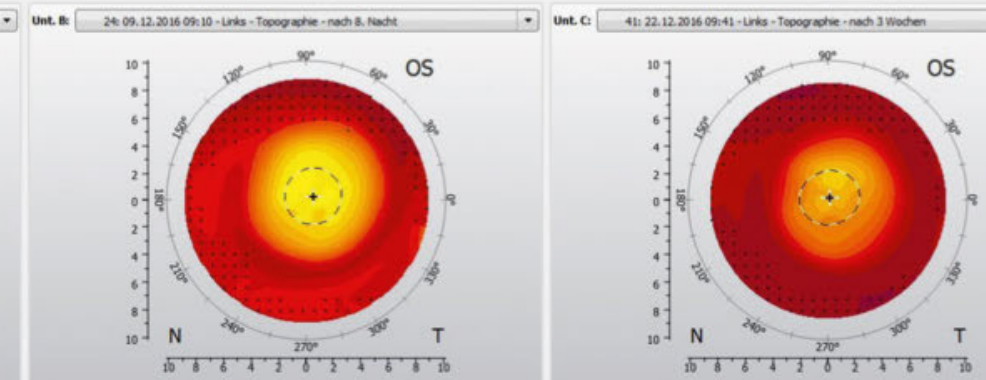

- Abb. 5 Prinzip der Orthokeratologielinsen.

a Schematische Darstellung der Orthokeratologie.

b Fluoresceinbild einer OK-Linse.

c Topografiebeispiel einer OK-Linse.

d OK-Topografiebild im Verlauf der Anpassung. 

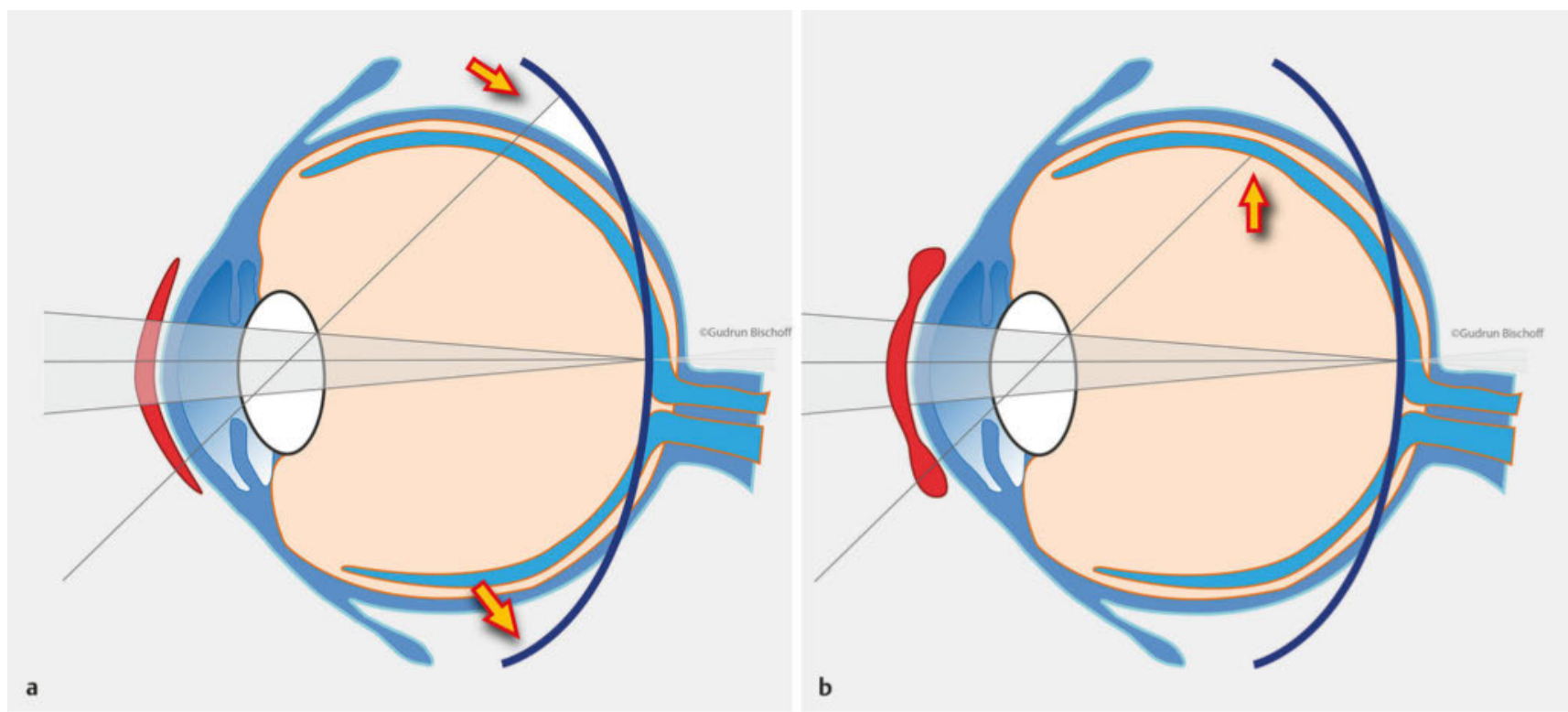

- Abb. 6 Myopie und Myopieprävention.

a Peripherer Fokus bei sphärischer Kontaktlinse.

b Peripherer Fokus bei Kontaktlinse mit peripherer Pluswirkung.

\section{Kontaktlinsen und Infektionsrisiko}

Insgesamt ist das Risiko einer mikrobiellen Keratitis klein, aber im Einzelfall bedrohlich für das Augenlicht. 75\% der keratitischen Ulzera sind kontaktlinsenassoziiert (s. a. Fallbeispiel 3). Prädisponierende Faktoren sind

- Hornhautoberflächendefekte durch schlechten Linsensitz,

- mangelnde Hygiene und

- unsaubere Umgebung [16].

Bei Kontaktlinsenträgern mit Zeichen einer Infektion wird die Therapie sofort mit einem topischen Breitbandantibiotikum begonnen. Gleichzeitig erfolgt eine Isolierung des Erregers ( $\triangleright$ Tab. 5) mittels Abstrich und Testung auf Sensibilität gegenüber gängigen Antibiotika [17].

Die Behandlung unterscheidet sich nicht von der üblichen augenärztlichen Behandlung.
\ Tab. 5 Die häufigsten Pathogene bei Kontaktlinsenproblemen.

\begin{tabular}{|l|l|}
\hline Erreger & Im Speziellen \\
\hline Bakterien & $\begin{array}{l}\text { Staphylococcus aureus } \\
\text { Pseudomonas aeruginosa } \\
\text { Streptococcus pneumoniae } \\
\text { Serratia spp. }\end{array}$ \\
\hline Pilze & $\begin{array}{l}\text { Fusarium } \\
\text { Aspergillus }\end{array}$ \\
\hline Hefen & Candida spp. \\
\hline Protozoen & Acanthamoeba spp. \\
\hline
\end{tabular}




\section{FALLBEISPIEL}

\section{Fall 3: Schmerzhafte Augen bei einem Kontaktlinsenträger}

Der 19 Jahre alte Patient kommt mit roten Augen in die Sprechstunde, er ist Kontaktlinsenträger - wie geht man vor?

\section{Gründliche Anamnese}

- Welche Linsen werden getragen?

- Welche Desinfektionsprodukte kommen wie zum Einsatz?

- Tragezeit der Kontaktlinsen?

\section{Diagnostik}

Nach der Anamnese erfolgen die Spaltlampeninspektion auch der Kontaktlinsen und ggf. ein Abstrich vom Auge und von der Flüssigkeit aus dem Linsenbehälter. Bei Verdacht auf bakterielle Infektion ist sofort mit einer Therapie zu beginnen [18].

Bei der Spaltlampenuntersuchung wird die Art der Rötung zugeordnet [19]. Klassisch für trockene Kontaktlinsenaugen und beginnende Kontaktlinsenkomplikationen ist die zirkuläre limbale violette Injektion. Periphere Infiltrate sind oft gesehen, aber nicht immer pathologisch [20]. Sie verursachen auch keine Rötung.

Die Symptome der beginnenden mikrobiellen Keratitis sind:

- Rötung des gesamten Bulbus,

- Schmerzen,

- Tränen,

- ggf. eitriges Sekret,

- Visusminderung.

Die Symptome variieren je nach Erreger, sind in der Regel aber gravierend ( $>$ Abb. 7).

\section{Weiteres Vorgehen}

Die Kontrollen der Therapie erfolgen kurzfristiger als bei Nichtlinsenträgern. Der Patient wird umfangreich aufgeklärt. Im ungünstigen Fall entwickelt sich innerhalb von Stunden ein schweres Ulkus mit einer nachfolgenden Endophthalmitis, dann muss sehr schnell gehandelt werden.

Falls eine Kontaktlinsentragepause angezeigt ist, dann nur so lange wie unbedingt nötig. In dieser Hinsicht zeigen die Kontaktlinsenträger wenig Compliance. Besser ist es, für besondere Gelegenheiten Tageslinsen mitzugeben, die dann nur Stunden getragen und danach sofort entsorgt werden.

\section{Fazit für die Praxis}

Durch das Tragen der Kontaktlinsen werden Schmerzen am Auge maskiert. Sie werden erst bemerkt nach Absetzen der Linse. Der Träger kommt oft sehr spät in die Sprechstunde, schnelles therapeutisches Handeln ist wichtig.
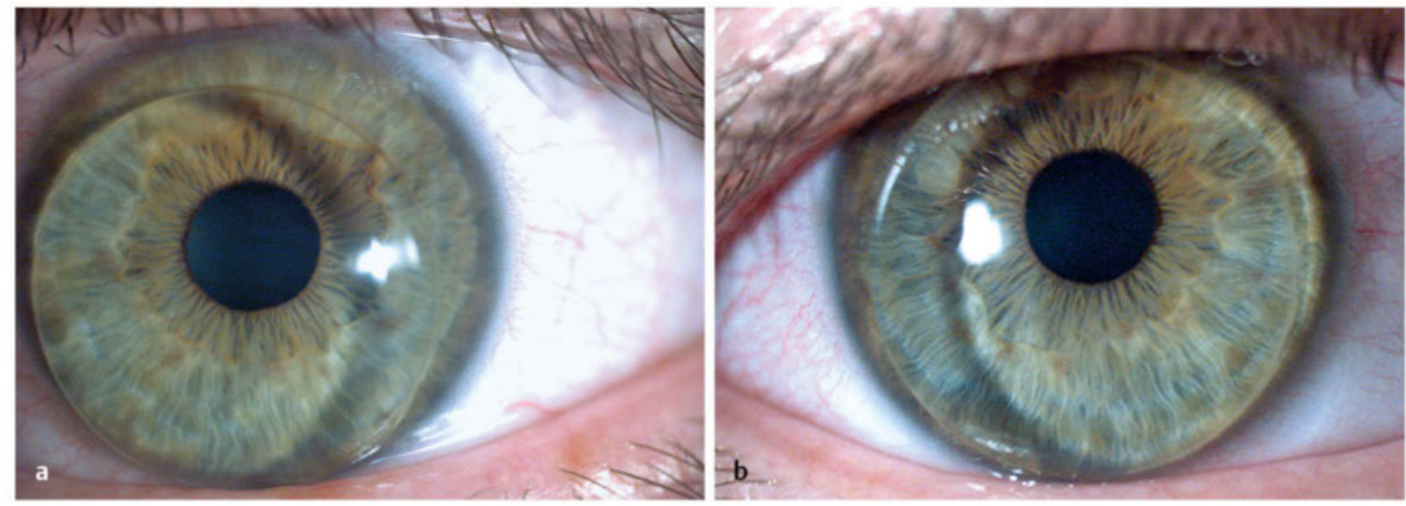

- Abb. 7 Fall 3.

a Limbäre Gefäßfülle.

b Kontaktlinsen mit klassischen roten Kontaktlinsenaugen. 


\section{Blick in die Zukunft}

Große Weltkonzerne haben die Kontaktlinsen für sich entdeckt, forschen und entwickeln. Über Mikrochips werden Messeinrichtungen in Kontaktlinsen eingebaut, man kann den Blutzucker messen, den Augendruck. Über die Nanotechnologie werden gezielt Medikamente aus der Kontaktlinse freigesetzt. Zur Prophylaxe von Infektionen experimentiert man mit keimtötenden Oberflächenbeschichtungen [21].

Kontaktlinsen, gleich ob kosmetisch und zum gelegentlichen Tragen, ob medizinisch in der differenzierten Therapie bei diversen Augenkrankheiten oder sogar als eigentlich refraktionsfremde Vehikel, haben einen festen Platz in der modernen Welt erobert.

\section{Zusammenfassung}

Im Beitrag werden Begriffe rund um die Kontaktlinsen erklärt.

Kontaktlinsen haben sich über die Jahre und Jahrzehnte sowohl in der Hilfsmittelversorgung als Refraktionsausgleich wie auch im medizinischen Einsatz in der Therapie von Hornhauterkrankungen einen festen Platz erobert. Ein Grund dafür ist die Vielfalt der Design- und Materialvarianten. Moderne Technologien in der Herstellung ermöglichen dies, für jede Fehlsichtigkeit kann die passende Kontaktlinse gefertigt werden.

Der Artikel weist auf besondere Indikationen der Kontaktlinsen hin: Komplikationen nach Lasik (Keratokonus) werden mit Kontaktlinsen behoben. Die Kontaktologie arbeitet in diesen Bereichen eng mit der Ophthalmochirurgie zusammen. Besondere Kontaktlinsenindikationen sind dazugekommen, wie die Presbyopiekontaktlinse, die Linsen zur Myopieprävention und die Ortho-K-Linsen, die sog. Nachtlinsen.

\section{KERNAUSSAGEN}

- Es gibt Kontaktlinsen in formstabiler Ausführung bis hin zu extrem flexiblen weichen Kontaktlinsen.

- Kontaktlinsen gibt es im konventionellen und im individuellen Bereich für alle Refraktionsfehler. Das ist möglich durch moderne Technologie.

- Kontaktlinsen sind für medizinische Indikationen zum Visuserhalt zwingend erforderlich, Beispiel Keratokonus.

- Die Verträglichkeit der Kontaktlinsen ist abhängig von vielen Faktoren, einmal von den Materialeigenschaften und dann von der physiologischen Interaktion mit dem vorderen Augenabschnitt.

- Formstabile Kontaktlinsen sind immer dann die erste Wahl, wenn es auf eine brillante Sehschärfe ankommt und ein Infektionsrisiko minimiert werden muss.

- Keratokonuskontaktlinsen sind in der Regel formstabil und in der Form auf den Konus abgestimmt. Die Anpassung wird EDV-gestützt erleichtert.

- Weiche Kontaktlinsen eignen sich hervorragend zum gelegentlichen Tragen, da sie kaum Gewöhnungszeit brauchen.

- Silikon-Hydrogel-Kontaktlinsen haben eine sehr hohe Sauerstoffdurchlässigkeit und sind biologisch verträglich.

- Silikon-Hydrogel-Linsen eignen sich als Therapieund Dauertragelinsen auch nach operativen Eingriffen im Bereich des vorderen Augenabschnittes.

- Presbyopiekontaktlinsen werden individuell angepasst und ausgewählt und erfordern einen verständigen Patienten.

Interessenkonflikt

Die Autorin gibt an, dass keine Interessenkonflikte vorliegen. 


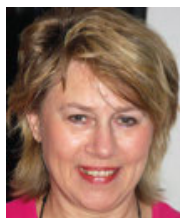

\section{Gudrun Bischoff}

Dr. med., Fachärztin für Augenheilkunde. Vorsitzende der Deutschen Augenärztlichen Kontaktlinsen Gesellschaft, Executive Secretary General und Past President des IMCLC, Direktor Medical Eye-Care Center Hamburg. Schwerpunkte dort: operative Versorgung des vorderen Augenabschnittes, Glaukombehandlung, pädiatrische Ophthalmologie mit Strabologie sowie Kontaktlinsenversorgung. Weiterhin: Vorsorge und die Therapie bei progredienter Myopie mit Kontaktlinsen plus additiver Verordnung von Atropin.

\section{Korrespondenzadresse}

\section{Dr. med. Gudrun Bischoff}

Augenpraxis und Augenklinik

Berner Heerweg 173-175

22159 Hamburg

gbischoff@medical-eye-care.de

Wissenschaftlich verantwortlich

gemäß Zertifizierungsbestimmungen

Wissenschaftlich verantwortlich gemäß Zertifizierungsbestimmungen für diesen Beitrag ist Dr. med. Gudrun Bischoff, Hamburg.

\section{Erstveröffentlichung}

Dieser Beitrag wurde erstveröffentlicht in: Klinische Monatsblätter für Augenheilkunde (doi:10.1055/a-0654-2441).

\section{Literatur}

[1] Leung BK, Bonanno JA, Radke CJ. Oxygen-deficient metabolism and corneal edema. Prog Retin Eye Res 2011; 30: 471 492. doi:10.1016/j.preteyeres.2011.07.001

[2] Bhamra TS, Tighe BJ. Mechanical properties of contact lenses: The contribution of measurement techniques and clinical feedback to 50 years of materials development. Cont Lens Anterior Eye 2017; 40: 70-81. doi:10.1016/j.clae.2016.11.005

[3] Kyle RA, Steensma DP, Shampo MA. Otto Wichterle-Inventor of the First Soft Contact Lenses. Mayo Clin Proc 2016; 91 : e45-e46. doi:10.1016/j.mayocp.2016.01.016

[4] Kim E, Saha M, Ehrmann K. Mechanical properties of contact lens materials. Eye Contact Lens 2017. doi:10.1097/ ICL.0000000000000442

[5] Moezzi AM, Fonn D, Varikooty J et al. Overnight corneal swelling with high and low powered silicone hydrogel lenses. J Optom 2015; 8: 19-26. doi:10.1016/j.optom.2014.03.002

[6] Long B, Schweizer $\mathrm{H}$, Bleshoy $\mathrm{H}$ et al. Expanding your use of silicone hydrogel contact lenses: using lotrafilcon A for daily wear. Eye Contact Lens 2009; 35: 59-64. doi:10.1097/ICL.0b013e318196ade7

[7] Yilmaz I, Ozcelik F, Basarir B et al. Clinical performance of toris $\mathrm{k}$ contact lens in patients with moderate to advanced keratoconus: a real life retrospective analysis. J Ophthalmol 2016; 2016: 2358901. doi:10.1155/2016/2358901
[8] Holden BA, Sankaridurg PR, Sweeney DF et al. Microbial keratitis in prospective studies of extended wear with disposable hydrogel contact lenses. Cornea 2005; 24: 156-161

[9] Perez-Prados R, Pinero DP, Perez-Cambrodi RJ et al. Soft multifocal simultaneous image contact lenses: a review. Clin Exp Optom 2016. doi:10.1111/cxo.12488

[10] Kohlhaas M. latrogene Keratektasie - eine Übersicht. Klin Monatsbl Augenheilkd 2015; 232: 765-772. doi:10.1055/s-00351545737

[11] Seiler TG, Fischinger I, Koller T et al. Superficial corneal crosslinking during laser in situ keratomileusis. J Cataract Refract Surg 2015; 41: 2165-2170. doi:10.1016/j.jcrs.2015.03.020

[12] Kasumovic SS, Pavljasevic S, Dacic-Lepara S et al. The results of corneal cross-linking in the treatment of keratoconus. Med Arch 2013: 67: 372-373

[13] Gatzioufas Z, Khine A, Elalfy M et al. Clinical outcomes after keraring implantation for keratoconus management in patients older than 40 years: a retrospective, interventional, cohort study. Ophthalmol Ther 2017. doi:10.1007/s40123-0170117-3

[14] Li X, Friedman IB, Medow NB et al. Update on orthokeratology in managing progressive myopia in children: efficacy, mechanisms, and concerns. J Pediatr Ophthalmol Strabismus 2017; 54: 142-148. doi:10.3928/01913913-20170106-01

[15] Walline J], Greiner KL, McVey ME et al. Multifocal contact lens myopia control. Optom Vis Sci 2013; 90: 1207-1214. doi:10.1097/OPX.0000000000000036

[16] Sauer A, Meyer N, Bourcier T et al. Risk factors for contact lensrelated microbial keratitis: a case-control multicenter study. Eye Contact Lens 2016; 42: 158-162. doi:10.1097| ICL.0000000000000180

[17] Konda N, Motukupally SR, Garg P et al. Microbial analyses of contact lens-associated microbial keratitis. Optom Vis Sci 2014; 91: 47-53. doi:10.1097/OPX.0000000000000082

[18] Fagan XJ, Jhanji V, Constantinou M et al. First contact diagnosis and management of contact lens-related complications. Int Ophthalmol 2012; 32: 321-327. doi:10.1007/s10792-0129563-z

[19] Efron N. Grading scales for contact lens complications. Ophthalmic Physiol Opt 1998; 18: 182-186

[20] Efron N, Morgan PB. Can subtypes of contact lens-associated corneal infiltrative events be clinically differentiated? Cornea 2006; 25: 540-544. doi:10.1097/01.ico.0000214219.67872. $3 c$

[21] Shayani Rad M, Khameneh B, Sabeti Z et al. Antibacterial Activity of Silver Nanoparticle-Loaded Soft Contact Lens Materials: The Effect of Monomer Composition. Curr Eye Res 2016; 41: 1286-1293. doi:10.3109/02713683.2015.1123726

\section{Bibliografie}

DOI https://doi.org/10.1055/s-0042-122988 Augenheilkunde up2date 2018; 8: 187-202 (c) Georg Thieme Verlag KG Stuttgart · New York ISSN 0023-2165 


\section{Punkte sammeln auf CME.thieme.de}

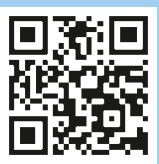

Sollten Sie Fragen zur Online-Teilnahme haben, finden Sie unter cme.thieme.de/hilfe eine ausführliche Anleitung. Wir wünschen viel Erfolg beim Beantworten der Fragen!

Unter eref.thieme.de/ZZWHPJE oder über den QR-Code kommen Sie direkt zum Artikel zur Eingabe der Antworten.

VNR 2760512018154652028

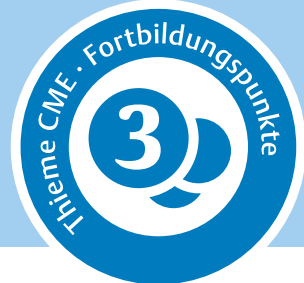

\section{Frage 1}

Was ist kein Kriterium für die Kontaktlinseneinordnung?
A Materialstabilität
B Sauerstoffdurchlässigkeit
C Größe
D Wassergehalt
E Desinfektionssystem

\section{Frage 2}

Welcher Parameter ist in hohem Maße verantwortlich für den Sitz der Linse?
A Durchmesser der Kontaktlinse
B Geometrie der Linsenrückfläche
C Zylinderstärke
D Gasdurchlässigkeit der Kontaktlinse
E Alter des Patienten

\section{Frage 3}

Einer der folgenden Befunde ist keine medizinische Kontaktlinsenindikation nach dem Hilfsmittelverzeichnis der gesetzlichen Krankenkassen. Welcher?
A hohe Myopie
B Astigmatismus von $1,5 \mathrm{dpt}$
C Irisanomalien
D Aniseikonie
E Aphakie

\section{Frage 4}

Welcher Begriff beschreibt nur eine Designvariante?
A Silikon-Hydrogel-Kontaktlinse
B semisklerale Kontaktlinse
C vorderflächentorische Linse
D Dauertragelinse
E weiche hochwasserhaltige Linse

\section{Frage 5}

Welches Design ist typisch für Presbyopiekontaktlinsen?
A korneale Linsen
B Minuslinsen
C hypergasdurchlässige Linsen
D segmentale Ringe unterschiedlicher Stärke
E Silikon-Hydrogellinsen

\section{Frage 6}

Welche Aussage ist richtig? Cross-Linking ...

A erwärmt die Hornhaut durch Bestrahlung.

B verschweißt die Hornhautfasern.

C vermehrt die Keratozytenzahl.

D wirkt biochemisch durch Vernetzung der Kollagenfibrillen.

E vermindert den Hornhautdurchmesser.

\section{Frage 7}

Welcher der folgenden Befunde ist eine gefürchtete späte Komplikation nach Lasik?
A schwankende Sehschärfe
B Kontaktlinsenunverträglichkeit
C sekundäre Ektasie
D torische Hornhauttopografie
E Augentropfenunverträglichkeit

\section{Frage 8}

Warum und wie wirkt die Orthokeratologie?

A durch die große Linse

B durch die hohe Sauerstoffdurchlässigkeit

C durch die lange Tragezeit

D durch eine Abflachung der Hornhaut im Zentrum von ca. $30 \mu \mathrm{m}$

E durch eine besondere innere Kurve

\section{- Weitere Fragen auf der folgenden Seite...}




\section{Punkte sammeln auf CME.thieme.de}

Fortsetzung...

\section{Frage 9}

Welche Kontaktlinsen werden speziell in der Myopieprävention eingesetzt?
A formstabile Linsen
B Minuslinsen
C torische Linsen
D individuelle Linsen
E Linsen mit peripherer Pluswirkung

\section{Frage 10}

Welcher Kontaktlinsentyp hat das kleinste Infektionsrisiko?
A Sklerallinsen
B rotationssymmetrische Linsen
C weiche Kontaktlinsen
D formstabile Kontaktlinsen
E Keratokonuslinsen 